\title{
Neuroprotective effect of edible bird's nest in chronic cerebral hypoperfusion induced neurodegeneration in rats
}

\author{
Rehab A. Ismaeil ${ }^{1}$, Chua Kien Hui ${ }^{2}$, Khairunisa Ahmad Affandi ${ }^{3}$, Batoul Alallam ${ }^{4}$, Wael Mohamed ${ }^{1,5}$, Mohd \\ Fadly Mohd Noor ${ }^{1}$
}

\begin{abstract}
'Department of Basic Medical Sciences, International Islamic University Malaysia, Kuantan 25200, Malaysia.
2Department of Physiology, University Kebangsaan, Kuala Lumpour 5600, Malaysia.

${ }^{3}$ Department of pathology and Laboratory Medicine, International Islamic University Malaysia, Kuantan 25200, Malaysia.

${ }^{4}$ Department of Pharmaceutical Technology, International Islamic University Malaysia, Kuantan 25200, Malaysia.

${ }^{5}$ Clinical Pharmacology department, Menoufia University, Shebin Elkom 32511, Egypt.
\end{abstract}

Correspondence to: Rehab A. Ismaeil, Department of Basic Medical Sciences, International Islamic University Malaysia, Kuantan-25200, Malaysia. E-mail: rehabpharma81@gmail.com

\begin{abstract}
How to cite this article: Ismaeil RA, Hui CK, Affandi KA, Alallam B, Mohamed W, Mohd Noor MF. Neuroprotective effect of edible bird's nest in chronic cerebral hypoperfusion induced neurodegeneration in rats. Neuroimmunol Neuroinflammation 2021;8:297-306. http://dx.doi.org/10.20517/2347-8659.2020.63.
\end{abstract}

Received: 16 Nov 2020 First Decision: 10 Dec 2020 Revised: 16 Dec 2020 Accepted: 21 Jan 2021 Available online: 21 Dec 2021

Academic Editor: Athanassios P. Kyritsis Copy Editor: Yue-Yue Zhang Production Editor: Xi-Jun Chen

\begin{abstract}
Aim: Alzheimer's disease (AD) is marked by insufficient blood supply to the brain, leading to progressive loss of memory and cognitive skills. The continuous reduction of cerebral blood flow resulting from chronic cerebral hypoperfusion leads to the overproduction of reactive oxygen species that triggers inflammation, causing cognitive decline. Till now, there is no cure for $A D$ and the only option is symptomatic treatment. The current study evaluates the effect of edible bird's nest (EBN) on hippocampal neurons, specifically in the CA1 hippocampal region, by using a rat model of chronic cerebral hypoperfusion-induced neuroinflammation.
\end{abstract}

Methods: Chronic cerebral hypoperfusion $(\mathrm{CCH})$ was induced by permanent bilateral common carotid artery occlusion (2VO) in rats to trigger the neuroinflammatory process. Rats were divided into 4 groups: sham, 2VO, and two $2 \mathrm{VO}$ groups treated with two different doses $(60 \mathrm{mg} / \mathrm{kg}, 120 \mathrm{mg} / \mathrm{kg}$ ) of EBN, which was administered daily by oral gavage. After 8 consecutive weeks, rats were euthanized and the hippocampi were examined histopathologically by counting viable neuronal cells and the levels of F2- Isoprostane in hippocampal tissue was measured by ELISA.

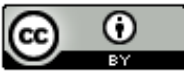

(C) The Author(s) 2021. Open Access This article is licensed under a Creative Commons Attribution 4.0 International License (https://creativecommons.org/licenses/by/4.0/), which permits unrestricted use, sharing, adaptation, distribution and reproduction in any medium or format, for any purpose, even commercially, as long as you give appropriate credit to the original author(s) and the source, provide a link to the Creative Commons license, and indicate if changes were made. 
Results: A significant decrease in neuronal cell death and a significant decline in F2-Isoprostane levels were observed in rats treated with EBN.

Conclusion: This is the first study to associate the neuroprotective effect of EBN in the prevention of dementia related to $A D$. EBN could be used as a complementary treatment to delay or treat dementia in AD patients.

Keywords: Alzheimer's disease, chronic cerebral hypoperfusion, edible bird's nest, F2-Isoprostanes

\section{INTRODUCTION}

The most prevalent neurodegenerative disorder that has dramatically increased over the last 60 years is Alzheimer's disease (AD). AD is a chronic disorder that starts years before the detection of any clinical cognitive decline, with memory loss and a decline in cerebral blood supply ${ }^{[1-3]}$. Chronic cerebral hypoperfusion $(\mathrm{CCH})$, along with various types of vascular insufficiency may initiate a cascade of events such as oxidative stress and inflammatory reactions that trigger vascular dementia ${ }^{[3-6]}$. As an established animal model for imitating the reduction in cerebral blood flow in human aging, permanent bilateral occlusion of common carotid arteries in rats (2-Vessel Occlusion, 2VO) has been introduced ${ }^{[7,8]}$. A continuous decrease in regional cerebral blood flow $(\mathrm{CBF})$ in different areas in the rat brain including the cerebral cortex and hippocampus causes neuronal dysfunction and impairs spatial learning, leading to memory deficits and dementia ${ }^{[3,7,9]}$. Previous studies of neuropathological changes caused by CCH focused on the hippocampus due to its essential role in learning and memory ${ }^{[8,10-12]}$. Furthermore, the hippocampal $\mathrm{CA} 1$ area is the most vulnerable in $\mathrm{CCH}^{[11,13,14]}$. In order to diagnose and treat the pathophysiological changes of $\mathrm{CCH}$ in an animal model, the detection of essential biomarkers would have a critical role. F2-Isoprostanes (F2-IsoPs) are prostaglandin-like molecules that are formed from the peroxidation of arachidonic acid. IsoPs are considered an accurate predictor of lipid peroxidation due to their biological stability $^{[13-16]}$.

Recent alternative medicines have underscored the neuroprotective and anti-inflammatory ability of edible bird's nest (EBN). EBN is one of the most valuable animal products eaten by humans, either for its therapeutic properties or as a delicacy in South East Asia ${ }^{[17,18]}$. It is formed naturally from salivary secretions of glutinous glycoprotein through the sublingual glands of swiftlets birds during their nesting and breeding season. The nest is built high on walls and the roofs of caves in the shape of a bowl over a period of 35 days. The solidified secretions enable the nest to carry the swiftlets and their eggs to remain attached to the wall during breeding season ${ }^{[17-19]}$. Researchers have investigated the therapeutic effects of EBN such as its anti-oxidative effect, anti-inflammatory effect, alleviation of osteoarthritis ${ }^{[20-22]}$, increased proliferation of the uterus, the enhancement of cell proliferation in corneal wound healing ${ }^{[23,24]}$ and its antiviral effect ${ }^{[25,26]}$. Moreover, it has the ability to reduce the risk of hypercoagulation caused by cardiovascular diseases associated with a high-fat $\operatorname{diet}^{[27]}$. Hou et al ${ }^{[28]}$ (2017) underscored the neuroprotective effect of EBN with elevated antioxidant activity in an ovariectomized rat model. EBN is also beneficial in reducing severity of neurodegenerative disorders due to its antioxidative effects on valuable bioactive components such as lactoferrin and ovotransferrin-like compounds ${ }^{[29]}$. Furthermore, sialic acid in EBN can enhance memory and improve cognitive function ${ }^{[30,31]}$. Additionally, EBN has epidermal growth factor-like activity, and is a rich source of proteins, carbohydrates, amino acids and minerals ${ }^{[1,32-34]}$. With such previous encouraging results, the current study investigated the neuroprotective and anti-inflammatory effects of EBN using a chronic cerebral hypoperfusion model. The occlusion of common carotid arteries triggers the formation of oxidative stress, causing inflammation in the brain hippocampus that then leads to neuronal death ${ }^{[6,11-14,35]}$. 


\section{METHODS}

\section{Animals}

The study was conducted using 24 male Sprague Dawley rats from a local vendor. We included only male rats to avoid possible confounding effect of the neuroprotective effect of female estrogen. Rats are a good animal model for establishing $2 \mathrm{VO}$ because they have a complete circle of Willis like humans and upon complete occlusion of one of the main branches of this circle, the body will react by forming new collaterals to bypass this obstruction to maintain cerebral blood flow ${ }^{[7]}$. The age of the rats was about 2 months and they weighed 200-250 g. The rats were fed with food pellets and tap water ad libitum at room temperature with a $12 \mathrm{~h} / 12 \mathrm{~h}$ dark/light cycle. Laboratory animal care was based on the guidelines and recommendations of the Integrated Centre for Research Animal Care and Use, IIUM and the Malaysian National Animal Welfare Foundation. After 1 week of acclimatization, rats were divided into four groups $(\mathrm{A}, \mathrm{B}, \mathrm{C}$, and $\mathrm{D})$ with 6 rats per group: sham group (A), which had surgery without permanent bilateral common carotid arteries occlusion; $2 \mathrm{VO}$ group (B) with permanent bilateral common carotid artery occlusion without any treatment; $2 \mathrm{VO}$ group that received a daily dose of $60 \mathrm{mg} / \mathrm{kg}$ EBN orally as treatment (C); and $2 \mathrm{VO}$ group that received a daily dose of $120 \mathrm{mg} / \mathrm{kg}$ EBN orally as treatment (D).

\section{$2 \mathrm{VO}$ procedure in rats}

Anesthetic agents, which consisted of ketamine $(90 \mathrm{mg} / \mathrm{kg})$ and xylazine $(20 \mathrm{mg} / \mathrm{kg})$ mixture were given via intraperitoneal injection. Ventral incisions $(2-3 \mathrm{~cm})$ were made in the neck area. The connective tissue and muscles layers were dissected to expose the common carotid arteries (CCAs) on both sides. Both CCAs were then carefully separated from the carotid sheath and vagus nerve, and then double ligated by sterilized silk sutures. Next, both arteries were cut between the two ligatures. Finally, the incision was closed with silk sutures and sterilized with povidone iodine $e^{[13,14]}$. The rat's body temperature was controlled during surgery and the recovery period under a heat lamp. Water and food were provided when rats became fully conscious. The Sham group followed similar procedures but without ligation of common carotid arteries. EBN treatment with oral gavage was started on the first postoperative day and was given for 8 consecutive weeks.

\section{EBN preparations}

EBN was provided by Glycofood Sdn Bhd, Balakong, Selangor, Malaysia in a dry powder form. One gram of EBN powder was dissolved in $100 \mathrm{~mL}$ of distilled water and heated for $45 \mathrm{~min}$ at $60{ }^{\circ} \mathrm{C}$. The EBN solution was brought to room temperature and administered by oral gavage depending on the rat's body weight. We administered two doses, $60 \mathrm{mg} / \mathrm{kg}$ and $120 \mathrm{mg} / \mathrm{kg}$ daily to Group C and D respectively by oral gavage for 8 consecutive weeks ${ }^{[24,28]}$. The treatment was given by oral gavage to mimic the mode of consumption of EBN in humans. Traditionally, EBN is prepared in the form of a soup and not taken as a medicine in tablets or injection form. Additionally, oral gavage was utilized to be sure the rats received the exact daily doses of $\mathrm{EBN}^{[30]}$.

\section{Histopathological study}

After the 8 th postoperative week, all rats were euthanized with inhalational ether. The rat brains were rapidly removed and immersed in ice-cold normal saline. The two hemispheres were separated and the hippocampi on both sides were carefully isolated. For histopathological examination, the left hippocampus was fixed in $10 \%$ neutral buffered formalin while the right hippocampus was washed with ice-cold water, dried and then stored in Eppendorf tubes at $-80{ }^{\circ} \mathrm{C}$ to measure the levels of F2-isoprostane by EnzymeLinked Immunosorbent Assay (ELISA). After the fixation and processing procedures, the left hippocampus was embedded in paraffin. Coronal sections of the dorsal hippocampus were taken within $1 \mathrm{~mm}$ of the horizontal distance from CA1, and sections of $5 \mu \mathrm{m}$ thickness were made with a rotatory microtome for staining with cresyl violet. Differences among the viable and non-viable neuronal cells were observed under a light microscope [model BX51TF (Olympus, Tokyo, Japan)] connected to a camera with 40x 

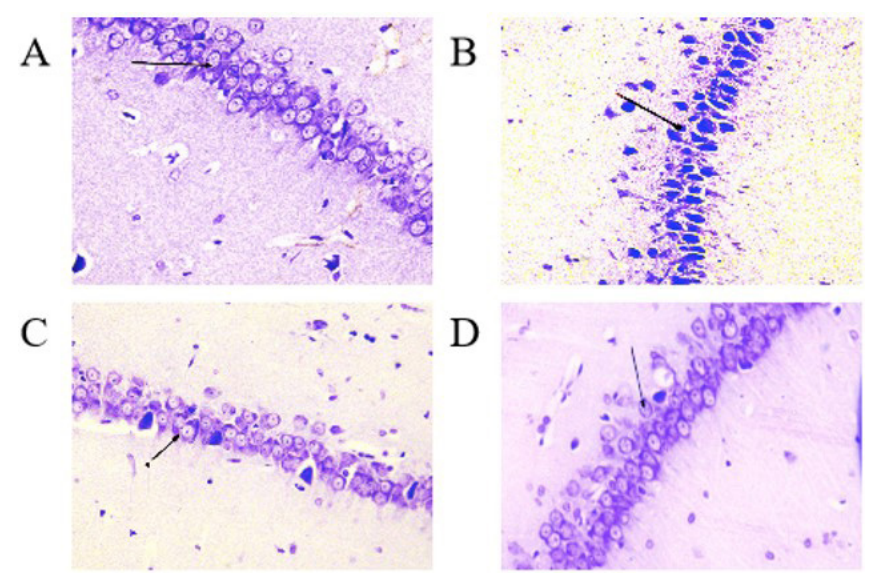

Figure 1. Cresyl violet stain shows histopathological changes in pyramidal cells within $1 \mathrm{~mm}$ of the hippocampus CA1 area in the Sham group (A), 2VO group (B), and EBN groups with different doses (C \& D) under light microscope. Arrows show the viable, well-defined neuronal cell with a distinct nucleus and fairly stained cytoplasm in A (Sham), C and D (EBN). In Group B (2VO), the arrow shows the pyknotic nuclei along with shrunken cytoplasm.

magnification. Viable neuronal cells (3 sections per animal) within $1 \mathrm{~mm}$ of the horizontal distance of the dorsal hippocampal CA1 were counted by using Image J software (USA).

\section{Biochemical analysis}

\section{Total protein assay}

Total protein concentration was determined by using bicinchonic acid protein Assay Kits, following the protocol recommended by the manufacturer (Thermo Scientific, USA). Bovine serum albumin was used as the standard with the absorbance of $562 \mathrm{~nm}$.

\section{F2-Isoprostane measurement}

To quantify the level of F2-Isoprostane in right hippocampi, tissues were minced and washed with phosphate buffer solution (PBS). The samples were then weighted and homogenized in PBS with a glass homogenizer (1 $\mathrm{gm}$ of tissue: $9 \mathrm{~mL}$ of PBS). Next, the samples were centrifuged for 5 mins at 500xg and the supernatant was collected. After that, ELISA was performed according to the protocol provided by the manufacturer (Elabscience-USA; ELISA kit of 8-epi-PGF-8-Epi-ProstaglandinF2Alpha).

\section{Statistical analysis}

All data were analyzed and presented as mean \pm SEM values with Minitab software version 17 (USA) and a $P$ value less than $0.05(P<0.05)$ was considered statistically significant. To compare the differences between means of various groups, One-way Analysis of Variance followed by Tukey post hoc test was conducted.

\section{RESULTS}

\section{Histopathological results}

Histopathological findings in the CA1 region of the hippocampus [Figure 1] revealed clear morphological differences between the Sham and 2VO groups. In the Sham group, the pyramidal cells have good density with a typical triangular shape, a well-defined cell membrane and a distinct nucleus with a fairly stained cytoplasm. However, the $2 \mathrm{VO}$ group revealed degenerated pyramidal cells with irregular cell membranes and dark pyknotic nuclei along with shrunken cytoplasm. On the other hand, the treated groups with different doses of EBN maintained the structures of the stratum pyramidal without significant neuronal cell loss. This indicated minimal neuronal damage compared to the untreated group (2VO group). Statistically, there was a significant increase in viable neuronal cells in the CA1 region in the Sham group compared to 


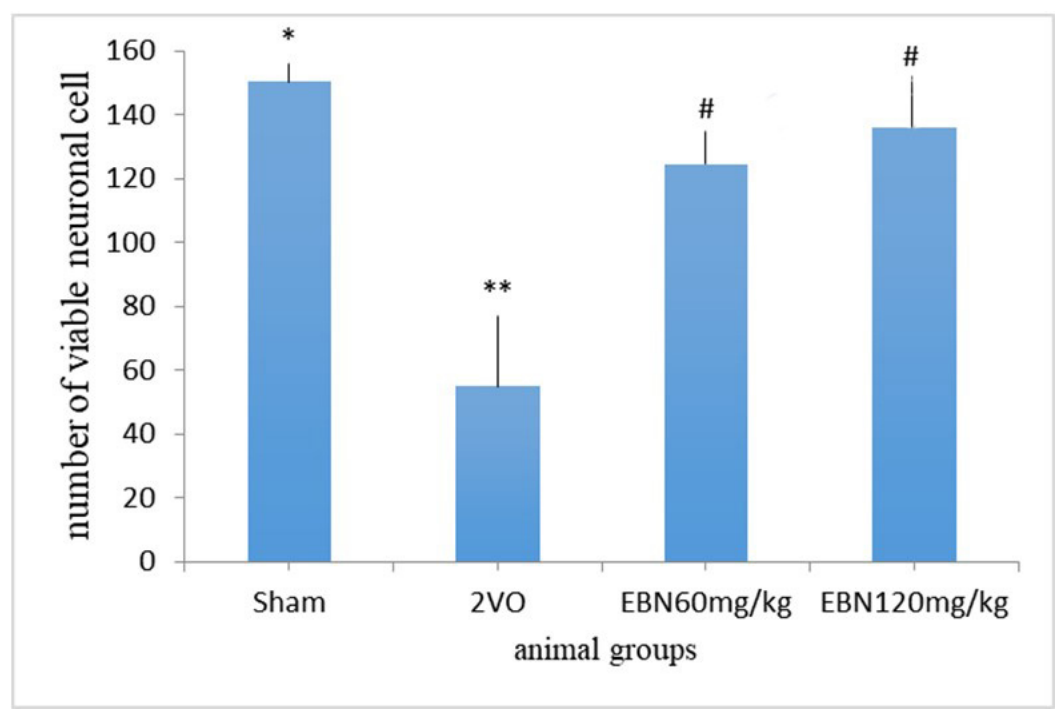

Figure 2. Bar chart indicates the number of viable neuronal cells in $1 \mathrm{~mm}$ of the hippocampus CA1 area of all groups. There was a decrease in the number of viable cells in the $2 \mathrm{VO}$ group compared to sham and the treated groups with EBN. Results are shown as mean \pm SEM. ${ }^{\star \star} P<0.001$ (Sham vs. 2VO); ${ }^{*} P<0.05$ (2VO vs. treated EBN groups); ${ }^{\star} P>0.05$ (Sham vs. treated EBN groups).

Table 1. Mean differences in neuronal cell count and F2-Isoprostane in all studied groups. Data are expressed as Mean \pm SEM

\begin{tabular}{lcccc}
\hline \multirow{2}{*}{ Parameters } & \multirow{2}{*}{ Sham } & \multirow{2}{*}{ 2Vo } & \multicolumn{2}{c}{ 2VO } \\
\cline { 3 - 5 } & & & EBN 60 $\mathbf{~ m g} / \mathbf{k g}$ & EBN 120 mg/kg \\
\hline Viable Neuronal cell & $154.4 \pm 0.477^{\star}$ & $54.8 \pm 6.11^{\star \star}$ & $124.6 \pm 1.45^{\#}$ & $136.2 \pm 1.22^{\#}$ \\
F2 IsoPs level in hippocampus CA1 & $0.5617 \pm 0.0915^{\star}$ & $1.436 \pm 0.141^{\star \star}$ & $0.8605 \pm 0.0705^{\#}$ & $0.7661 \pm 0.0388^{\#}$ \\
\hline
\end{tabular}

This table demonstrates the difference in mean value of viable neuronal cells and the mean value of F2-isoprostane in all the study groups with ${ }^{\star \star} P<0.001$ (Sham vs. $2 \mathrm{VO}$ ); ${ }^{\#} P<0.05\left[2 \mathrm{VO}\right.$ vs. $2 \mathrm{VO}+\left(\mathrm{EBN} 60 \mathrm{mg} / \mathrm{kg} \& \mathrm{EBN} 120 \mathrm{mg} / \mathrm{kg}\right.$ )]; ${ }^{\star} P>0.05$ (Sham vs. EBN treated groups).

the $2 \mathrm{VO}$ groups $(P<0.001)$. The count of viable neuronal cells in the Sham and $2 \mathrm{VO}$ groups were $(154.4 \pm 0.477)$ and $(54.8 \pm 6.11)$ respectively. Conversely, there was a significant increase in viable neuronal cells in the treated groups of $2 \mathrm{VO}$ with different doses of EBN as compared to the untreated $2 \mathrm{VO}$ group. The number of viable neuronal cells in EBN $(60 \mathrm{mg} / \mathrm{kg})$ treated group was $(124.6 \pm 1.45)$ and $\mathrm{EBN}(120 \mathrm{mg} / \mathrm{kg})$ treated group was $(136.2 \pm 1.22)$ with a p-value less than $0.05(P<0.05)$ compared to the untreated group. Interestingly, there was no significant difference between both EBN treated groups compared to the Sham group [Figure 2 and Table1].

\section{Biochemical results}

\section{F2-Isoprostane level measurement}

As shown in Figure 3 and Table 1, the level of F2-IsoPs per mg protein in the untreated 2 VO group (1.436 $\pm 0.141 \mathrm{pg} / \mathrm{mg}$ protein) was significantly higher $(P<0.001)$ compared to the Sham group $(0.5617 \pm 0.0915$ $\mathrm{pg} / \mathrm{mg}$ protein). On the other hand, the hippocampal F2-IsoPs levels were significantly reduced in the $2 \mathrm{VO}$ group treated with $60 \mathrm{mg} / \mathrm{kg}$ of EBN $(0.8605 \pm 0.0705 \mathrm{pg} / \mathrm{mg}$ protein $)$ and $120 \mathrm{mg} / \mathrm{kg}$ of EBN $(0.7661 \pm$ $0.0388 \mathrm{pg} / \mathrm{mg}$ protein) respectively when compared to the untreated $2 \mathrm{VO}$ group. On further analysis, the F2-IsoPs levels in both EBN treated groups showed no significant difference when compared to the Sham group.

\section{DISCUSSION}

$\mathrm{AD}$ is considered one of the biggest socio-economic problems that can cost billions of dollars. Despite enormous advancements in drug research, the prevalence of $\mathrm{AD}$ worldwide continues to be on the rise due 


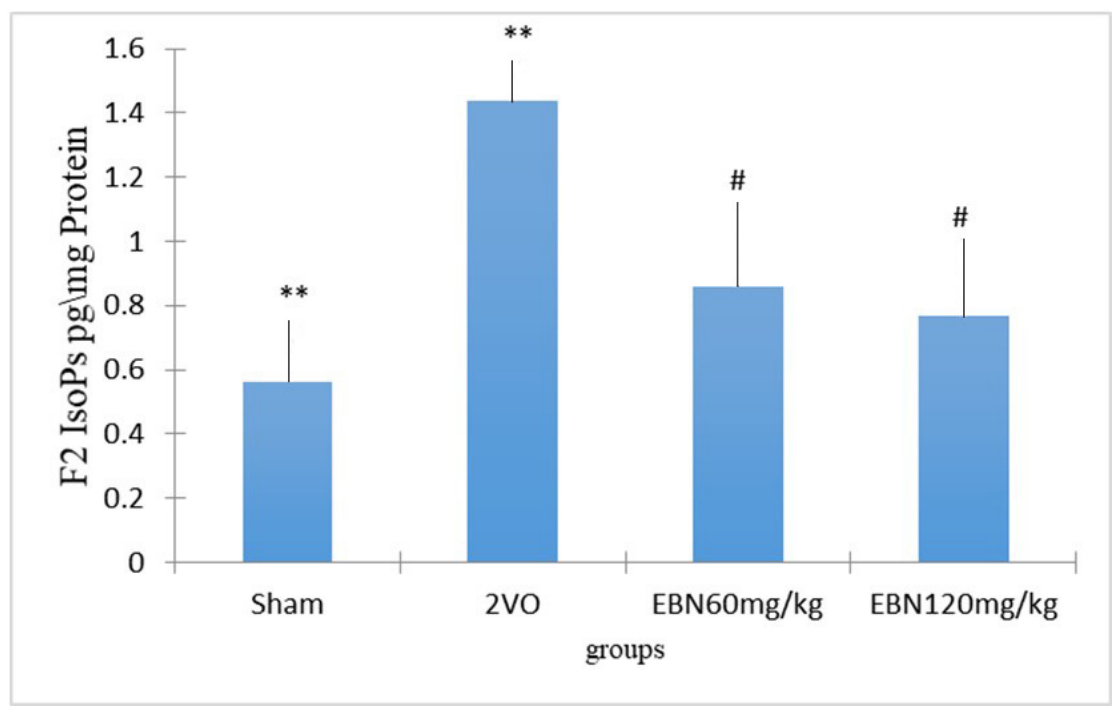

Figure 3. Bar chart shows the F2-Isoprostane level in the hippocampus of different groups. The results showed an increased level of F2Isoprosten in the $2 \mathrm{VO}$ group compared to Sham, while the level declined in the EBN treated groups. There was no significant difference between the Sham and treated groups with EBN. Results represented as mean $\pm \mathrm{SEM}{ }^{\star \star} P<0.001$ (2VO vs. Sham). \#P<0.05[2VO vs. $(2 \mathrm{vo}+60 \mathrm{mg} / \mathrm{kg}$ EBN and $2 \mathrm{vo}+120 \mathrm{mg} / \mathrm{kg}$ EBN) ]. $P>0.05$ (Sham vs. EBN treated group).

to its associated long-term complications. The challenges in $\mathrm{AD}$ research today include the discovery of strategies for early identification and new therapies for disease prevention and cure $e^{[1,36,37]}$.

Chronic cerebral hypoperfusion can be induced by $2 \mathrm{VO}$ with reduction in cerebral blood flow in a rat model. This occlusion is irreversible and permanent, leading to pathophysiological changes that increase the incidence of $\mathrm{AD}$ and vascular dementia in animal models ${ }^{[1,38]}$. The most vulnerable area of the brain affected by chronic cerebral hypoperfusion is the hippocampal CA1 that leads to learning and memory impairment ${ }^{[13-14]}$. Several studies support the notion that oxidative stress is a significant contributor in AD development ${ }^{[39-41]}$. The lack of reactive oxygen species protection in the aging brain leads to progression of the disease. Several pathological mechanisms are activated by cerebral hypoperfusion such as oxidative stress and inflammation that culminate in neuronal cell death ${ }^{[12,38,42]}$. In chronic cerebral hypoperfusion, there is an increased level of free radicals within the brain due to imbalance between oxidants and antioxidants ${ }^{[14,40]}$. Furthermore, the inflammatory response triggered by oxidative stress causes the release of cytokines, chemokines, activated microglia and astrocytes, which then release more pro-inflammatory cytokines that cause neuronal cell death ${ }^{[4,11,14,43]}$.

Several researchers have also indicated that cognitive functions are linked to the number of neurons in the hippocampal CA1 area. Therefore, neuroprotection can lead to positive outcomes in cognitive abilities via several natural products ${ }^{[6,8,9,11,40]}$ as adjuncts to the available pharmacological treatments for AD. The treatment strategy by using natural antioxidants and anti-inflammatory drugs may be helpful in scavenging the free radicals to prolong the survival of neuronal cells in cerebral hypoperfusion ${ }^{[11-14,40-44]}$.

In the current study, we induced long term brain ischemia in male rats by permanent occlusion of the common carotid arteries bilaterally, which triggers the formation of oxidative stress in the brain hippocampus. We used relatively young rats to tolerate the $2 \mathrm{VO}$ surgery, which is an invasive operation. The main aim of the current study is to investigate the neuroprotective effect of EBN and not its treatment potential. Therefore, young rats were used to extrapolate our data to younger individuals with risk factors that may predispose to developing dementia later. Also, we can mirror our data to cognitively impaired individuals to improve their cognitive abilities and prevent further deterioration. We started the treatment 
a day after surgery to protect neurons from damage as the CBF decreases dramatically after occlusion (acute phase), and stop after 8 weeks as CBF returns to normal due to the establishment of new collaterals. Our findings show that $2 \mathrm{VO}$ rats have decreased number of viable neuronal cells and increased levels of lipid peroxidation F2- isoprostane within the hippocampal CA1 area. Interestingly, after treatment with EBN for eight consecutive weeks, there was an improvement in pathological changes of neuronal cells associated with an increase in viable neuronal cell count in the CA1 hippocampal area. In addition, the decreased levels of oxidative stress in the hippocampus compared to untreated groups is in agreement with previous studies that demonstrate the antioxidant and anti-inflammatory effects of EBN contribute towards the reduction of neuronal damage and oxidative stress ${ }^{[45,46]}$. Our results are consistent with several studies that have demonstrated the health benefits of EBN as a safe, natural food with anti-oxidant and antiinflammatory functions. A study done in 2015 on estrogen deficiency in a rat model demonstrated that EBN was able to reduce neurological dysfunctions in the hippocampus and frontal cortex with an increase in antioxidant enzyme activity and decrease in oxidative stress ${ }^{[47]}$. Furthermore, EBN has also been shown to enhance cognitive function ${ }^{[28]}$ and is able to improve the learning skills of offspring in mice by increasing the number of viable neuronal cells in the hippocampal CA1, CA2 and CA3 regions ${ }^{[48]}$. Careena et al. ${ }^{[30]}$ demonstrated in 2018 the anti-inflammatory impact of sialic acid in EBN. It improves learning and memory in Wistar rats by inhibiting neuro-inflammation and oxidative stress. Therefore, EBN may be suggested as a new-alternative treatment in neurodegenerative disorders such as $\mathrm{AD}$ and Parkinsonism that are triggered by oxidative stress ${ }^{[49]}$. All these findings indicate that EBN could reduce the neurodegeneration and cognitive deterioration induced by inflammation and oxidative stress.

In conclusion our results show that $\mathrm{CCH}$, which was triggered by $2 \mathrm{VO}$, causes a reduction in viable neurons in the hippocampal CA1 region. This damage could be ameliorated by the administration of EBN, which exhibits a neuroprotective effect via increasing the viable neuronal cell count, and a decline in oxidative stress levels. Our results underscored the possible effects of EBN in delaying the progression of dementia in $\mathrm{AD}$ patients if used early in the disease. Moreover, EBN may be used as an adjuvant treatment with any cognitive enhancer drugs (CED) in patients with $\mathrm{AD}$ or dementia to minimize its dose and hence, CED side effects while delaying the development of drug resistance. Furthermore, EBN can be taken prophylactically as a daily dietary supplement in the elderly to protect brain neurons against the aging process. Overall, EBN is a natural and safe supplement that could be used prophylactically for a long time.

\section{Study limitations and perspectives}

The current study is the first of its kind that investigated the possible neuroprotective effects of EBN. The current results underscored the potential effect of EBN in delaying the progression of AD dementia, if taken early before the onset of the disease. Our study has some methodological limitations. It would be ideal to use a validated anti-dementia drug as a positive control (e.g., Methylphenidate) to compare with the effects of EBN. Also, our sample size of six was relatively small. We plan to replicate the current study in the future using a bigger sample size. It is interesting to note that our data analysis are preliminary and requires further investigation with adding behavioral testing to assess the learning and memory effects of EBN. In future studies, it will be effective if we studied both gene and protein expression in the hippocampus and cortex in the brain, in addition to behavioral studies and clinical trials. Further, using other animal models e.g., mice, or zebra fish may be of great importance to investigate the neuroprotective effect of EBN in different species with different genetic profiles.

\section{DECLARATIONS}

\section{Acknowledgments}

The authors would like to express their gratitude to Glycofood Sdn Bhd Company, Balakong, Selangor, Malaysia for providing the EBN pure powder free of charge. 


\section{Authors' contributions}

Concept, study design, performing all experiment and procedures, data generation, data analysis, and writing manuscript: Ismaeil RA

Hypothesis, study design, data generation, data analysis, and writing manuscrip: Mohd Noor MF, Mohamed W

Data analysis, critical review and editing manuscript: Hui CK, Affandi KA

Data generation, data analysis and editing manuscript: Alallam B

\section{Availability of data and materials}

Not applicable.

\section{Financial support and sponsorship}

None.

\section{Conflicts of interest}

All authors declared that there are no conflicts of interest.

\section{Ethical approval and consent to participate}

IIUM/ IACUC-2019(15).

\section{Consent for publication}

Not applicable.

\section{Copyright}

(C) The Author(s) 2021.

\section{REFERENCES}

1. Alzheimer's Association. 2020 Alzheimer's disease facts and figures. Available from https://www.alz.org/media/Documents/alzheimersfacts-and-figures.pdf. [Last accessed on 1 Feb 2021]

2. de la Torre J. The vascular hypothesis of Alzheimer's disease: a key to preclinical prediction of dementia using neuroimaging. $J$ Alzheimers Dis 2018;63:35-52.

3. Duncombe J, Kitamura A, Hase Y, Ihara M, Kalaria RN, Horsburgh K. Chronic cerebral hypoperfusion: a key mechanism leading to vascular cognitive impairment and dementia. Closing the translational gap between rodent models and human vascular cognitive impairment and dementia. Clin Sci (Lond) 2017;131:2451-68.

4. Navarro V, Sanchez-Mejias E, Jimenez S, et al. Microglia in Alzheimer's disease: activated, dysfunctional or degenerative. Front Aging Neurosci 2018; 10:140.

5. Sarlus H, Heneka MT. Microglia in Alzheimer's disease. J Clin Invest 2017;127:3240-9.

6. Wang N, He J, Pan C, et al. Resveratrol activates autophagy via the AKT/mTOR signaling pathway to improve cognitive dysfunction in rats with chronic cerebral hypoperfusion. Front Neurosci 2019;13:859.

7. Farkas E, Luiten PG, Bari F. Permanent, bilateral common carotid artery occlusion in the rat: a model for chronic cerebral hypoperfusionrelated neurodegenerative diseases. Brain Res Rev 2007;54:162-80.

8. Wang DP, Chen SH, Wang D, et al. Neuroprotective effects of andrographolide on chronic cerebral hypoperfusion-induced hippocampal neuronal damage in rats possibly via PTEN/AKT signaling pathway. Acta Histochem 2020;122:151514.

9. Yao ZH, Yao XL, Zhang SF, Hu JC, Zhang Y. Tripchlorolide may improve spatial cognition dysfunction and synaptic plasticity after chronic cerebral hypoperfusion. Neural Plast 2019;2019:2158285.

10. Wang DP, Yin H, Lin Q, et al. Andrographolide enhances hippocampal BDNF signaling and suppresses neuronal apoptosis, astroglial activation, neuroinflammation, and spatial memory deficits in a rat model of chronic cerebral hypoperfusion. Naunyn Schmiedebergs Arch Pharmacol 2019;392:1277-84.

11. Pirmoradi Z, Yadegari M, Moradi A, Khojasteh F, Zare Mehrjerdi F. Effect of berberine chloride on caspase-3 dependent apoptosis and antioxidant capacity in the hippocampus of the chronic cerebral hypoperfusion rat model. Iran J Basic Med Sci 2019;22:154-9.

12. Hei Y, Chen R, Yi X, Long Q, Gao D, Liu W. HMGB1 neutralization attenuates hippocampal neuronal death and cognitive impairment in rats with chronic cerebral hypoperfusion via suppressing inflammatory responses and oxidative stress. Neuroscience 2018;383:150-9.

13. Azimi M, Ashour AE, Fuaat AA, Mohamed WMY. Neuroprotective effects of coenzyme Q10 and vitamin-E in chronic cerebral hypoperfusion-induced neurodegeneration in rats. Int J Nutr 2020;10:35-42. 
14. Mohamed WMY, Sayeed S, Saxena AK, Oothuman P. Oxidative stress status and neuroprotection of tocotrienols in chronic cerebral hypoperfusion-induced neurodegeneration rat animal model. Int J Nutr 2018;8:47-52.

15. Arikawa AY, Samavat H, Gross M, Kurzer MS. Plasma $F_{2}$-isoprostanes are positively associated with glycemic load, but inversely associated with dietary polyunsaturated fatty acids and insoluble fiber in postmenopausal women. J Nutr 2017;147:1693-9.

16. Milne GL, Dai Q, Roberts LJ 2nd. The isoprostanes-25 years later. Biochim Biophys Acta 2015;1851:433-45.

17. Ma F, Liu D. Sketch of the edible bird's nest and its important bioactivities. Food Research International 2012;48:559-67.

18. Marcone MF. Characterization of the edible bird's nest the "Caviar of the East". Food Research International 2005;38:1125-34.

19. Ramji MFS, Koon LC, Rahman MA. Roosting and nest-building behaviour of the white-nest swiftlet Aerodramus fuciphagus (Thunberg) (Aves: Apodidae) in farmed colonies. Raffl es Bull Zool 2013;29:225-35.

20. Chua KH, Lee TH, Nagandran K, et al. Edible Bird's nest extract as a chondro-protective agent for human chondrocytes isolated from osteoarthritic knee: in vitro study. BMC Complement Altern Med 2013;13:19.

21. Matsukawa N, Matsumoto M, Bukawa W, et al. Improvement of bone strength and dermal thickness due to dietary edible bird's nest extract in ovariectomized rats. Biosci Biotechnol Biochem 2011;75:590-2.

22. Hu Q, Li G, Yao, et al. Edible bird's nest enhances antioxidant capacity and increases lifespan in drosophila melanogaster. Cell Mol Biol (Noisyle-grand) 2016; 62:116-22.

23. Zainal Abidin F, Hui CK, Luan NS, Mohd Ramli ES, Hun LT, Abd Ghafar N. Effects of edible bird's nest (EBN) on cultured rabbit corneal keratocytes. BMC Complement Altern Med 2011;11:94.

24. Albishtue AA, Yimer N, Zakaria MZA, et al. Edible bird's nest impact on rats' uterine histomorphology, expressions of genes of growth factors and proliferating cell nuclear antigen, and oxidative stress level. Vet World 2018;11:71-9.

25. Guo CT, Takahashi T, Bukawa W, et al. Edible bird's nest extract inhibits influenza virus infection. Antiviral Res 2006;70:140-6.

26. Haghani A, Mehrbod P, Safi N, Kadir FA, Omar AR, Ideris A. Edible bird's nest modulate intracellular molecular pathways of influenza A virus infected cells. BMC Complement Altern Med 2017;17:22.

27. Yida Z, Imam MU, Ismail M, Ismail N, Hou Z. Edible bird's nest attenuates procoagulation effects of high-fat diet in rats. Drug Des Devel Ther 2015;9:3951-9.

28. Hou Z, He P, Imam MU, et al. Edible Bird's Nest prevents menopause-related memory and cognitive decline in rats via increased hippocampal Sirtuin-1 expression. Oxid Med Cell Longev 2017;2017:7205082.

29. Hou Z, Imam MU, Ismail M, et al. Lactoferrin and ovotransferrin contribute toward antioxidative effects of Edible Bird's Nest against hydrogen peroxide-induced oxidative stress in human SH-SY5Y cells. Biosci Biotechnol Biochem 2015;79:1570-8.

30. Careena S, Sani D, Tan SN, et al. Effect of Edible Bird's Nest extract on lipopolysaccharide-induced impairment of learning and memory in wistar rats. Evid Based Complement Alternat Med 2018;2018:9318789.

31. Pozsgay V, Jennings H, Kasper DL. 4,8-anhydro-N-acetylneuraminic acid. Isolation from edible bird's nest and structure determination. Eur J Biochem 1987;162:445-50.

32. Kong Y, Keung W, Yip T, Ko K, Tsao S, Ng M. Evidence that epidermal growth factor is present in swiftlet's (Collocalia) nest. Comparative Biochemistry and Physiology Part B: Comparative Biochemistry 1987;87:221-6.

33. Norhayati MK Jr, Azman O, Wan Nazaimoon W. Preliminary study of the nutritional content of malaysian edible bird's nest. Malays $J$ Nutr 2010;16:389-96.

34. Babji AS, Etty Syarmila IK, Nur 'Aliah D, et al. Assessment on bioactive components of hydrolysed edible bird nest. Int Food Res $J$ 2018;25:1936-41.

35. Saxena AK, Abdul-Majeed SS, Gurtu S, Mohamed WM. Investigation of redox status in chronic cerebral hypoperfusion-induced neurodegeneration in rats. Appl Transl Genom 2015;5:30-2.

36. Alzheimer's Disease International. World Alzheimer Report 2015. The global impact of dementia: an analysis of prevalence, incidence, cost and trends. Available online from: https://www.alzint.org/u/WorldAlzheimerReport2015.pdf. [Last accessed on 1 Feb 2021]

37. Cazarim Mde S, Moriguti JC, Ogunjimi AT, Pereira LR. Perspectives for treating Alzheimer's disease: a review on promising pharmacological substances. Sao Paulo Med J 2016;134:342-54.

38. Zhang D, Xiao Y, Lv P, et al. Edaravone attenuates oxidative stress induced by chronic cerebral hypoperfusion injury: role of ERK/Nrf2/ HO-1 signaling pathway. Neurol Res 2018;40:1-10.

39. Luca M, Luca A, Calandra C. The role of oxidative damage in the pathogenesis and progression of Alzheimer's Disease and vascular dementia. Oxid Med Cell Longev 2015;2015:504678.

40. Fanoudi S, Alavi MS, Hosseini M, Sadeghnia HR. Nigella sativa and thymoquinone attenuate oxidative stress and cognitive impairment following cerebral hypoperfusion in rats. Metab Brain Dis 2019;34:1001-10.

41. Zhao X, Fang J, Li S, et al. Artemisinin attenuated Hydrogen Peroxide $\left(\mathrm{H}_{2} \mathrm{O}_{2}\right)$-induced oxidative injury in SH-SY5Y and hippocampal neurons via the activation of AMPK pathway. Int J Mol Sci 2019;20:2680.

42. Zhang J, Liu Y, Zheng Y, et al. TREM-2-p38 MAPK signaling regulates neuroinflammation during chronic cerebral hypoperfusion combined with diabetes mellitus. J Neuroinflammation 2020;17:2.

43. Zulfiqar S, Garg P, Nieweg K. Contribution of astrocytes to metabolic dysfunction in the Alzheimer's disease brain. Biol Chem 2019;400:1113-27.

44. Zeng Q, Huang Z, Zhang J, et al. 3'-Daidzein sulfonate sodium protects against chronic cerebral hypoperfusion-mediated cognitive impairment and hippocampal damage via activity-regulated cytoskeleton-associated protein upregulation. Front Neurosci 2019;13:104.

45. Yida Z, Imam MU, Ismail M, et al. Edible Bird's Nest attenuates high fat diet-induced oxidative stress and inflammation via regulation of hepatic antioxidant and inflammatory genes. BMC Complement Altern Med 2015;15:310. 
46. Yida Z, Imam MU, Ismail M. In vitro bioaccessibility and antioxidant properties of edible bird's nest following simulated human gastrointestinal digestion. BMC Complement Altern Med 2014;14:468.

47. Zhiping H, Imam MU, Ismail M, et al. Effects of edible bird's nest on hippocampal and cortical neurodegeneration in ovariectomized rats. Food Funct 2015;6:1701-11.

48. Xie Y, Zeng H, Huang Z, et al. Effect of maternal administration of Edible Bird's Nest on the learning and memory abilities of suckling offspring in mice. Neural Plast 2018;2018:7697261.

49. Yew MY, Koh RY, Chye SM, Othman I, Ng KY. Edible bird's nest ameliorates oxidative stress-induced apoptosis in SH-SY5Y human neuroblastoma cells. BMC Complement Altern Med 2014;14:391. 